\title{
MicroRNA-552 promotes hepatocellular carcinoma progression by downregulating WIF1
}

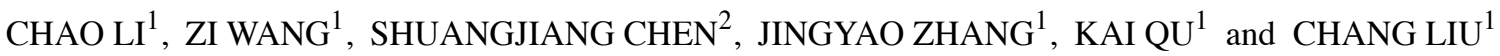 \\ ${ }^{1}$ Department of Hepatobiliary Surgery, The First Affiliated Hospital of Xi'an Jiaotong University, Xi'an, Shaanxi 710061; \\ ${ }^{2}$ Department of General Surgery, Ankang People's Hospital, Ankang, Shaanxi 725000, P.R. China
}

Received March 22, 2018; Accepted September 4, 2018

DOI: 10.3892/ijmm.2018.3882

\begin{abstract}
MicroRNAs (miRNAs/miRs) are involved in the metastasis of hepatocellular carcinoma (HCC). In the present study, it was demonstrated that miR-552 was upregulated in HCC tissues. High miR-552 expression was associated with malignant clinicopathological features and decreased survival rates. The in vitro results indicated that miR-552 overexpression promoted migration, invasion and epithelial-mesenchymal transition in Hep3B cells. However, the knockdown of miR-552 inhibited its oncogenic roles in Huh-7 cells. Additionally, Wnt inhibitory factor 1 (WIF1) was demonstrated to be a direct target of miR-552 in Hep3B and Huh-7 cells. Additional experiments identified that miR-552 promotes $\beta$-catenin expression by increasing the phosphorylation of GSK3 $\beta$ at Ser9. In conclusion, the results suggested that miR-552 may promote HCC progression by blocking WIF1-mediated GSK $3 \beta$ dephosphorylation. miR-552 may be a biomarker for predicting the outcomes of patients with HCC.
\end{abstract}

\section{Introduction}

MicroRNAs (miRNAs/miRs) are a class of RNAs measuring $<25$ bp in length that do not have protein-coding functions (1). Previous studies have demonstrated that miRNAs may regulate cell proliferation, invasion, immune response and metabolism in physiological and pathophysiological processes by binding to the 3 ' untranslated region (3'-UTR) of targeting mRNAs to decrease their expression (2).

Hepatocellular carcinoma (HCC) is a serious health burden worldwide, particularly in Asia and Africa (3). Although advanced therapeutic methods have been implemented in previous years, clinical data indicate that patients suffering

Correspondence to: Professor Chang Liu, Department of Hepatobiliary Surgery, The First Affiliated Hospital of Xi'an Jiaotong University, 277 Yanta West Road, Xi'an, Shaanxi 710061, P.R. China

E-mail: liuchangdoctor@163.com

Key words: microRNA-552, hepatocellular carcinoma, invasion, epithelial-mesenchymal transition, Wnt inhibitory factor 1 with metastasis usually have poor prognoses (4). Therefore, the control of tumor metastasis is an important strategy to improve the prognosis of HCC. At present, there is increasing evidence that miRNAs serve critical roles in HCC metastasis $(5,6)$. For example, the downregulation of miR-1271 in HCC tissues was associated with venous infiltration, and ectopic expression of miR-1271 may inhibit invasion and lung metastatic nodules formation (7).

miR-552, a novel cancer-associated miRNA, has been studied primarily in colorectal cancer (CRC) (8). Functionally, miR-552 was demonstrated to increase CRC cell migration and invasion abilities (9). However, the functions of miR-552 in the metastasis of HCC and detailed mechanisms associated with epithelial-mesenchymal transition (EMT), which is an important mechanism for cell migration and invasion (10), remains unclear.

The present study identified that miR-552 was upregulated in HCC. High expression of miR-552 predicted poor overall survival and disease-free survival in a 3-year follow-up period. In vitro, miR-552 was demonstrated to increase migration, invasion and EMT by targeting Wnt inhibitory factor 1 (WIF1). Additionally, glycogen synthase kinase $3 \beta$ (GSK3 $\beta$ )/ $\beta$-catenin signaling activation was confirmed as an important mechanism for the oncogenic functions of miR-552.

\section{Materials and methods}

Ethical review. The use of clinical samples was approved by the Ethics Committee of First Affiliated Hospital of Xi'an Jiaotong University (Xi'an, China) according to the 1975 Declaration of Helsinki. Written informed consent was obtained and signed by each patient enrolled in the present study.

Clinical samples and cell lines. A total of $76 \mathrm{HCC}$ tissues and matched tumor-adjacent tissues were collected from 53 male patients and 23 female patients who received surgery from the Department of Hepatobiliary Surgery, First Affiliated Hospital of Xi'an Jiaotong University between January 2010 and January 2014. Tissues were stored in liquid nitrogen. Human immortalized normal hepatocyte cell line (LO2) and HCC cell lines (Huh-7, MHCC97-L, Hep3B, MHCC97-H) were purchased from the Institute of Biochemistry and Cell Biology, Chinese Academy of Sciences (Shanghai, China) and stored at the Centre for Translational Medicine at the First 
Affiliated Hospital of Xi'an Jiaotong University. Cells were cultured with Dulbecco's modified Eagle's medium (DMEM; Gibco; Thermo Fisher Scientific, Inc., Waltham, MA, USA) supplemented with $10 \%$ fetal bovine serum (FBS; Gibco; Thermo Fisher Scientific, Inc.) and 1\% V/V penicillin/streptomycin (Sigma-Aldrich; Merck KGaA, Darmstadt, Germany) in a humidified atmosphere containing $5 \% \mathrm{CO}_{2}$ at $37^{\circ} \mathrm{C}$.

Cell transfection. miR-552 mimics (miR10003215-1-5; miR-552), miR-552 inhibitor (miR20003215-1-5; miR-552-inhi), negative control mimics (miR01201-1-5; miR-ctrl), negative control inhibitor (miR02201-1-5; miR-ctrl-inhi), WIF1 small interfering (si)RNA (stB0009885; si-WIF1) and negative control siRNA (siN05815122147-1-5; si-ctrl) were purchased from RiboBio Co. (Guangzhou, China). Negative control vectors (Vec-ctrl) and WIF1 plasmids (SC119176) were obtained from OriGene (Rockville, MD, USA). A total of $10 \mu \mathrm{g}$ mimics, inhibitors, siRNAs or plasmids were used to transfect HCC cells using Lipofectamine 3000 (Invitrogen; Thermo Fisher Scientific, Inc.) according to the manufacturer's protocol. After $48 \mathrm{~h}$ transfection, cells were harvested for further experiments.

Reverse transcription-quantitative polymerase chain reaction $(R T-q P C R)$. TRIzol ${ }^{\circledR}$ reagent (Invitrogen; Thermo Fisher Scientific, Inc.) was used to isolate total RNA from tissues and cultured cells. RT-PCR and qPCR were performed using the Superscript III Reverse Transcriptase kit (Invitrogen; Thermo Fisher Scientific, Inc.) and iTaq Universal SYBR-Green Supermix kit (Bio-Rad Laboratories, Inc., Hercules, CA, USA), respectively. The Bulge-Loop miR-552 RT-qPCR Primer Set (Guangzhou RiboBio, Co., Ltd., Guangzhou, China) was applied to measure the miR-552 expression. The primer sequences were as follows: WIF1 forward, 5'- GTGTGAAAT CAGCAAATGCC-3' and reverse, 5'- GTCTTCCATGCCAAC CTTCT-3'; and GAPDH forward, 5'-CCAGGGCTGCTTTTA ACTCT-3' and reverse, 5'-GGACTCCACGACGTACTCA-3'). The thermocycling conditions were as follows: Initial denaturation at $50^{\circ} \mathrm{C}$ for $2 \mathrm{~min}$ and enzyme denaturation at $95^{\circ} \mathrm{C}$ for $10 \mathrm{~min}$, followed by 40 cycles: Denaturation at $95^{\circ} \mathrm{C}$ for $15 \mathrm{sec}$, annealing at $60^{\circ} \mathrm{C}$ for $30 \mathrm{sec}$, elongation at $70^{\circ} \mathrm{C}$ for $1 \mathrm{~min}$, and a final extension at $72^{\circ} \mathrm{C}$ for $10 \mathrm{~min}$. The $2^{-\Delta \Delta \mathrm{Cq}}$ method (11) was used to calculate the relative gene expression normalized by GAPDH.

Western blot analysis. Cells were lysed with radioimmunoprecipitation assay lysis buffer (Xi'an Hut Biotechnology Co., Ltd., Xian, China) and quantified with a BCA protein assay kit II (cat.no. 5000002; Bio-Rad Laboratories, Inc.). Protein samples $(40 \mu \mathrm{g})$ were separated by $10 \%$ SDS-PAGE gel and transferred onto a nitrocellulose membrane (Invitrogen; Thermo Fisher Scientific, Inc.) using Bio-Rad tank blotting system (Bio-Rad Laboratories, Inc.). Membranes were blocked with 5\% bovine serum albumin (Sigma-Aldrich; Merck KGaA) with 1X TBST (TBS with $0.1 \%$ Tween-20) for $2 \mathrm{~h}$ at room temperature. The membranes were incubated with the appropriate primary antibodies for a 1:1,000 dilution overnight at $4^{\circ} \mathrm{C}$. Horseradish peroxidase (HRP)-conjugated secondary antibodies at a 1:2,000 dilution were used to incubate the membranes for $1 \mathrm{~h}$ at room temperature subsequent to washing them with TBST (TBS with $0.1 \%$ Tween-20) 3 times for $10 \mathrm{~min}$. The targeting proteins on the membrane were visualized with enhanced chemiluminescent (ECL) reagents (EMD Millipore; Billerica, MA, USA). Membranes incubated with ECL reagents were wrapped in plastic wrap box and exposed to x-ray film. Exposed x-ray films were then scanned to a black-and-white figure by a scanner (LiDE 220, Canon, Inc., Tokyo, Japan). The intensity of every protein band was calculated using ImageJ software [V1.8.0_112; National Institutes of Health (NIH), Bethesda, MD, USA]. The corresponding GAPDH intensity was used to calculate the relative intensity of the bands.

The primary antibodies used for the western blot analysis were as follows: WIF1 (cat. no. ab155101) was purchased from Abcam (Cambridge, MA, USA); epithelial cadherin (E-cadherin; cat. no. 3195), vimentin (cat. no. 3932), GSK3 $\beta$ (cat. no. 12456) and phosphorylated GSK3 $\beta$ (cat. no. 5558) antibodies were purchased from Cell Signaling Technology, Inc. (Danvers, MA, USA); and matrix metalloproteinase (MMP)-2 (cat. no. sc-13594) and GAPDH (cat. no. sc-47724) antibodies were purchased from Santa Cruz Biotechnology, Inc. (Dallas, Texas, USA).

Immunohistochemical staining. Paraformaldehyde-fixed paraffin-embedded HCC tissue sections were prepared by Xi'an Hut Biotechnology Co., Ltd. and used for immunohistochemical staining. WIF1 primary antibodies were diluted in PBS at 1:100 to label the WIF1 at $4^{\circ} \mathrm{C}$ overnight. Biotinylated goat anti-rabbit secondary antibodies (cat. no. SP-9001; ZSGB-BIO; OriGene Technologies, Inc., Beijing, China) were used to detect the primary antibodies. Complexes were detected by HRP-streptavidin conjugates (ZSGB-BIO; OriGene Technologies, Inc.) and visualized with DAB (ZSGB-BIO; OriGene Technologies, Inc.). The final immunohistochemical staining scores were calculated as described previously (12).

Wound healing assay. Following transfection, cells were cultured in 6-well plates and grown to $80-90 \%$ confluence. A wound across the middle of the well was created by a $200 \mu 1$ sterile pipette tip. Subsequently, cells were cultured with serum-reduced (2\% FBS v/v) DMEM in a humidified $5 \% \mathrm{CO}_{2}$ incubator at $37^{\circ} \mathrm{C}$ for 0 and $48 \mathrm{~h}$. Images were captured with a phase-contrast microscope at a magnification of $\mathrm{x} 40$ and analyzed by Image J software (V1.8.0_112; NIH).

Transwell assay. Transwell inserts (Nalge Nunc, Penfield, NY, USA) were coated with Matrigel (BD Biosciences, Franklin Lakes, NJ, USA) at $1 \mathrm{mg} / \mathrm{ml}$ on the upper layer and incubated at $37^{\circ} \mathrm{C}$ for $30 \mathrm{~min}$. Briefly, $3 \times 10^{4}$ cells were seeded into the upper chamber with serum-reduced (2\% FBS v/v) DMEM. A total of $750 \mu \mathrm{l}$ DMEM containing $10 \%$ FBS was added into the lower chamber. Cells were then incubated in a humidified $5 \% \mathrm{CO}_{2}$ incubator at $37^{\circ} \mathrm{C}$ for $24 \mathrm{~h}$. Chambers were fixed in $4 \%$ paraformaldehyde for $5 \mathrm{~min}$ at room temperature and then stained with $0.3 \%$ crystal violet dye for $10 \mathrm{~min}$. Non-invading cells were removed by a cotton swab. Invaded cells were counted under a light microscope at a magnification of x 200 .

Dual-luciferase reporter assay. TargetScan (http://www. targetscan.org/) and MiRanda (http://www.microrna.org) were used to predict the target of miR-552. The predicted wild-type WIF1 3'-UTR sequence corresponding with miR-552 and 
the mutant 3'-UTR sequence were synthesized and inserted into the pEZX-MT06 plasmid vector (GeneCopoeia, Inc., Rockville, MD, USA). Recombinant constructs, miR-552 mimics or miR-552 inhibitors were co-transfected into the Huh-7 and Hep3B cells using Lipofectamine 3000 (Invitrogen; Thermo Fisher Scientific, Inc.) according to the manufacturer's protocol. After $48 \mathrm{~h}$, the cells were harvested, and luciferase activity was measured using the Luc-Pair Duo-Luciferase Assay kit 2.0 (GeneCopoeia, Inc.). Firefly luciferase activity was normalized to Renilla luciferase activity.

XAV939 cell treatment. XAV939 (cat. no. X3004) was purchased from Sigma-Aldrich; Merck KGaA, and dissolved in dimethyl sulfoxide (DMSO; Sigma-Aldrich; Merck KGaA). A total of $20 \times 10^{4}$ cells were seeded into 6 -well plate and cultured at $37^{\circ} \mathrm{C}$ overnight. XAV939 was used to treat cells at a final concentration of $5 \mu \mathrm{M}$ for $24 \mathrm{~h}$. DMSO was used to treat the cells as a control.

Statistical analysis. Continuous variables are presented as the mean \pm standard deviation. Statistical analysis was performed using SPSS version 21.0 software (SPSS Inc.; IBM Corp., Armonk, NY, USA) or GraphPad PRISM 5 software (GraphPad Software, Inc., La Jolla, CA, USA). Correlations between miR-552 and clinical features were analyzed by Pearson's $\chi^{2}$ test. The correlation between miR-552 and WIF1 expression was analyzed by Spearman correlation analysis. The difference between two groups was analyzed using an unpaired Student t-test. One-way analysis of variance was used to compare the data among multiple groups, and the Bonferroni method was used for the post-hoc test. Survival analysis was performed using a Kaplan-Meier curve and log-rank test. $\mathrm{P}<0.05$ was considered to indicated a statistically significant difference.

\section{Results}

Expression of miR-552 is upregulated in HCC tissues and predicts poor prognosis. The expression of miR-552 in human tissues was analyzed by RT-qPCR. A significant increase in miR-552 in HCC tissues compared with the adjacent non-tumor tissues was identified (Fig. $1 \mathrm{~A} ; \mathrm{P}<0.01$ ). The clinical significance of miR-552 in patients with $\mathrm{HCC}$ was then investigated. A total of two subgroups, miR-552 high expression group ( $\geq$ mean, $n=50)$ and miR-552 low expression group ( $<$ mean, $\mathrm{n}=26$ ), in the cohort were divided according to the mean level of miR-552. In Table I, the results from Pearson's $\chi^{2}$ test indicated that high expression of miR-552 was associated with venous invasion $(\mathrm{P}=0.035)$ and advanced Tumor Node Metastasis (13) stage $(\mathrm{III}+\mathrm{IV} ; \mathrm{P}=0.024)$, indicating that tumors with high miR-552 expression levels may be more aggressive. Therefore, the difference in miR-552 expression between aggressive tissues (tumors with intrahepatic metastasis, bile duct invasion or venous infiltration) and non-aggressive HCC tissues was determined. As hypothesized, compared with non-aggressive HCC tissues, miR-552 was significantly upregulated in aggressive HCC tissues (Fig. 1B; $\mathrm{P}<0.05$ ).

Furthermore, Kaplan-Meier survival curves were used to describe the prognostic value of miR-552 in HCC. Patients with high miR-552 expression exhibited a decrease in overall
Table I. Clinical significance of miR-552 in hepatocellular carcinoma.

\begin{tabular}{|c|c|c|c|c|}
\hline \multirow[b]{2}{*}{ Clinical features } & \multicolumn{2}{|c|}{$\begin{array}{l}\text { miR-552 } \\
\text { expression }\end{array}$} & \multirow[b]{2}{*}{$\chi^{2}$} & \multirow[b]{2}{*}{ P-value } \\
\hline & $\begin{array}{l}\text { High } \\
(n=50)\end{array}$ & $\begin{array}{c}\text { Low } \\
(n=26)\end{array}$ & & \\
\hline Sex & & & 1.259 & 0.262 \\
\hline Male & 37 & 16 & & \\
\hline Female & 13 & 10 & & \\
\hline Age, years & & & 1.284 & 0.257 \\
\hline$<60$ & 22 & 15 & & \\
\hline$\geq 60$ & 28 & 11 & & \\
\hline Hepatitis B infection & & & 1.404 & 0.236 \\
\hline Absent & 11 & 9 & & \\
\hline Present & 39 & 17 & & \\
\hline Tumor size, $\mathrm{cm}$ & & & 2.606 & 0.107 \\
\hline$<5$ & 27 & 19 & & \\
\hline$\geq 5$ & 23 & 7 & & \\
\hline Liver cirrhosis & & & 0.866 & 0.352 \\
\hline Absent & 14 & 10 & & \\
\hline Present & 36 & 16 & & \\
\hline $\begin{array}{l}\text { Serum } \alpha \text { fetoprotein } \\
\text { level, } \mathrm{ng} / \mathrm{ml}\end{array}$ & & & 2.155 & 0.142 \\
\hline$<400$ & 20 & 15 & & \\
\hline$\geq 400$ & 30 & 11 & & \\
\hline Venous invasion & & & 4.451 & 0.035 \\
\hline Absent & 33 & 23 & & \\
\hline Present & 17 & 3 & & \\
\hline $\begin{array}{l}\text { Edmondson-Steiner } \\
\text { grading }\end{array}$ & & & 1.748 & 0.186 \\
\hline $\mathrm{I}+\mathrm{II}$ & 19 & 14 & & \\
\hline $\mathrm{III}+\mathrm{IV}$ & 31 & 12 & & \\
\hline $\begin{array}{l}\text { Tumor Node Metastasis } \\
\text { stage }\end{array}$ & & & 5.077 & 0.024 \\
\hline $\mathrm{I}+\mathrm{II}$ & 21 & 18 & & \\
\hline III+IV & 29 & 8 & & \\
\hline
\end{tabular}

survival [Fig. 1C, hazard ratio $(\mathrm{HR})=2.541 ; 95 \%$ confidence interval (CI) 1.442-4.478; $\mathrm{P}=0.001]$ and disease-free survival (Fig. 1D; HR=2.435, 95\% CI 1.390-4.263; $\mathrm{P}=0.002$ ). These data suggest that miR-552 is a potential biomarker for the clinical outcome of patients with HCC.

miR-552 promotes the migration, invasion and EMT of HCC cells. Next, qPCR was used to demonstrate that miR-552 was upregulated in all HCC cell lines with the exception of Hep3B (Fig. 2A; $\mathrm{P}<0.05$ ). miR-552 expression was then silenced in Huh-7 cells (Fig. 2B; $\mathrm{P}<0.01$ ) and overexpressed in Hep3B cells (Fig. 2C; $\mathrm{P}<0.001$ )

Wound-healing and Transwell assays were used to determine the migration and invasion, respectively, in vitro. In 

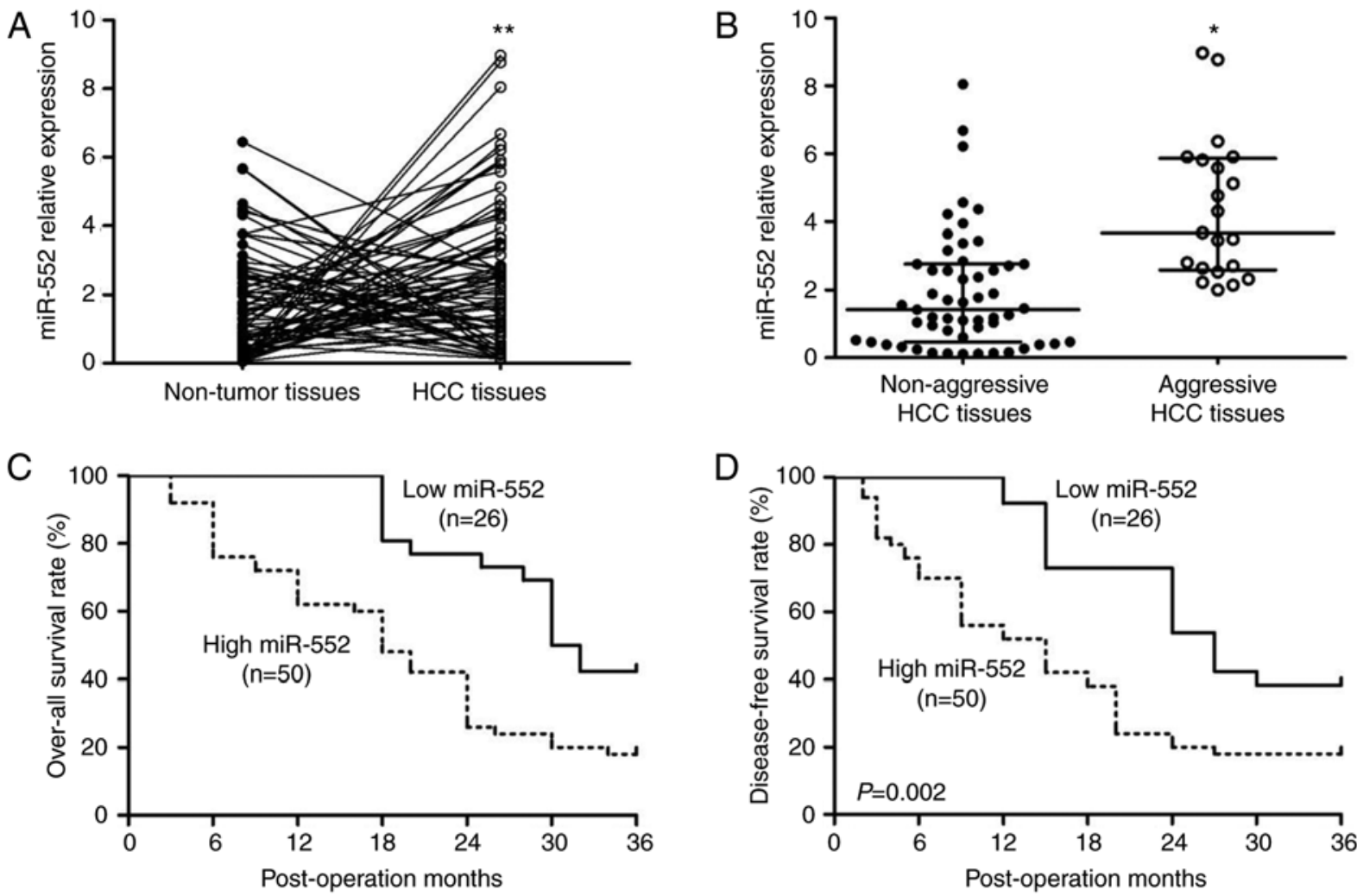

Figure 1. Upregulation of miR-552 predicts worse prognosis in patients with HCC. (A) The expression of miR-552 was upregulated in 76 HCC tissues. (B) HCC tissues with aggressive clinical features exhibited higher miR-552 expression levels compared with non-aggressive tissues. The patients in the miR-552 high expression group exhibited (C) a lower overall survival rate and (D) a shorter disease-free survival time. ${ }^{*} \mathrm{P}<0.05$ and ${ }^{* *} \mathrm{P}<0.01$. miR, microRNA; HCC, hepatocellular carcinoma.
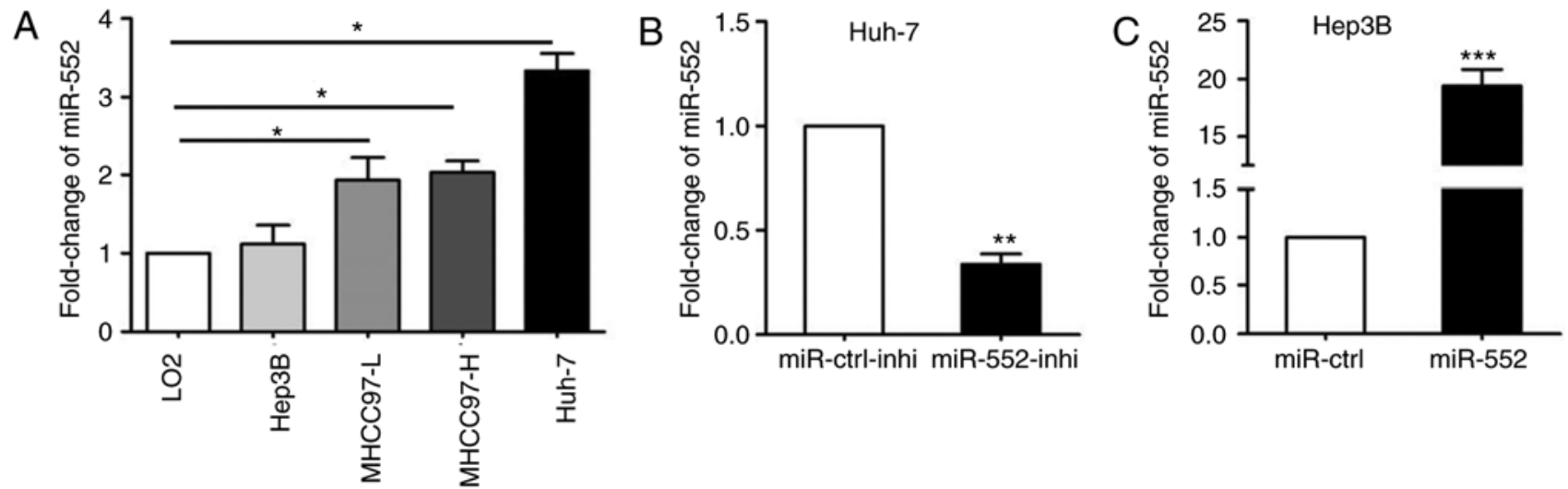

Figure 2. Transfection of miR-552 inhibitors and mimics in HCC cells. (A) miR-552 expression was measured in the human immortalized normal hepatocyte LO2 cell line and 4 hepatocellular carcinoma Huh-7, MHCC97-L, Hep3B, MHCC97-H cell lines by reverse transcription quantitative polymerase chain reaction. (B) The expression of miR-552 was knocked down by transfecting its inhibitors into Huh-7 cells. (C) Transfection of miR-552 mimics increased miR-552 expression in Hep3B cells. ${ }^{*} \mathrm{P}<0.05,{ }^{* *} \mathrm{P}<0.01$ and ${ }^{* * * *} \mathrm{P}<0.001$. miR, microRNA; ctrl, control; inhi, inhibitor.

was observed that downregulation of miR-552 decreased cell migration (Fig. 3A; $\mathrm{P}<0.05$ ) and invasion (Fig. 3B; $\mathrm{P}<0.05$ ) in Huh-7 cells. Conversely, the overexpression of miR-552 significantly promoted cell migration (Fig. 3C; $\mathrm{P}<0.05$ ) and invasion (Fig. 3D, $\mathrm{P}<0.05$ ) in Hep3B cells. This result indicated that miR-552 may be associated with tumor metastasis in $\mathrm{HCC}$.

Tumor migration and invasion are complex processes and are regulated by several cellular processes, including a loss of cell-cell adhesion that is frequently accompanied by the downregulation of E-cadherin and upregulation of vimentin. The western blot analysis results of the present study indicated that knockdown of miR-552 increased E-cadherin expression and decreased vimentin expression in Huh-7 cells (Fig. 3E; P<0.05), whereas upregulation of miR-552 inhibited E-cadherin, but induced vimentin expression in Hep3B cells (Fig. 3F; $\mathrm{P}<0.05$ ). These results indicate that miR-552 possesses marked oncogenic functions in HCC cells. 
A
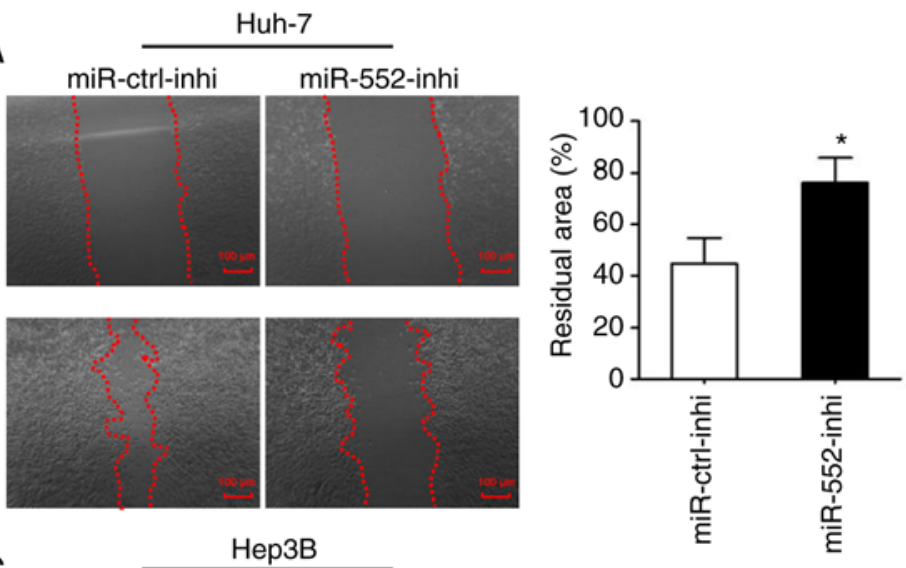

B
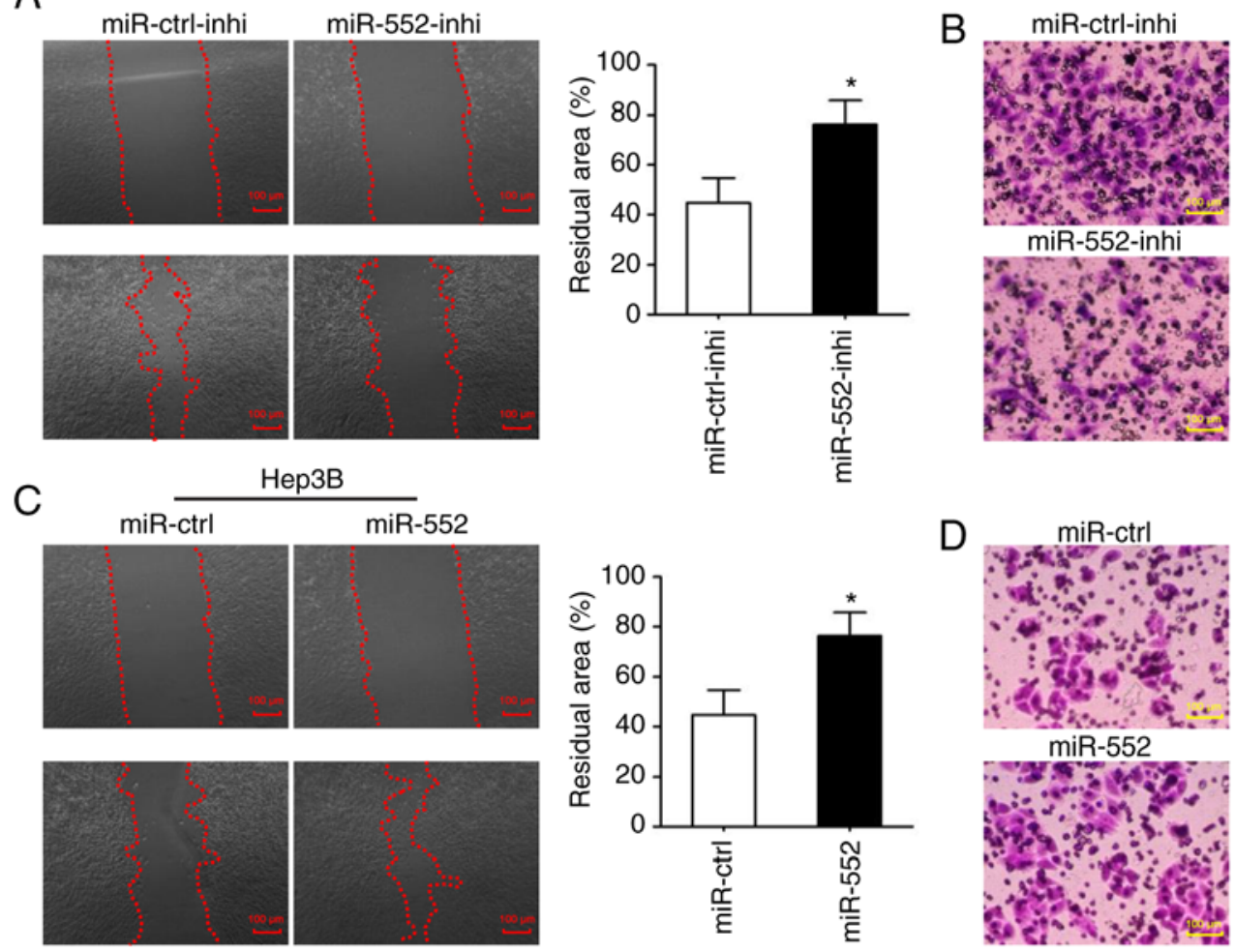
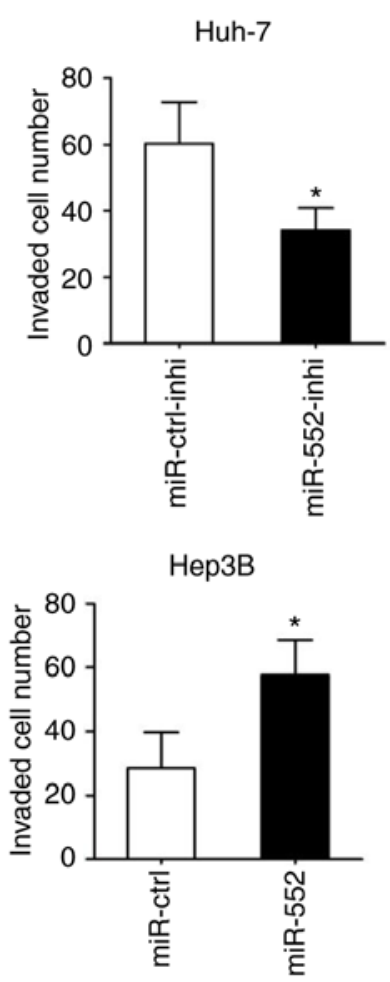

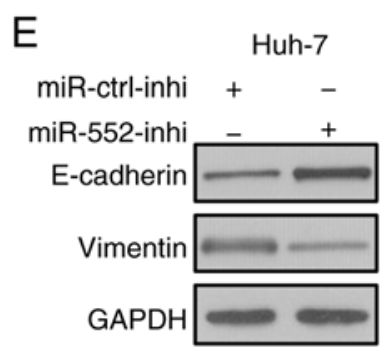

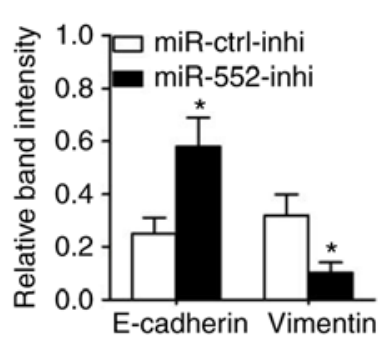
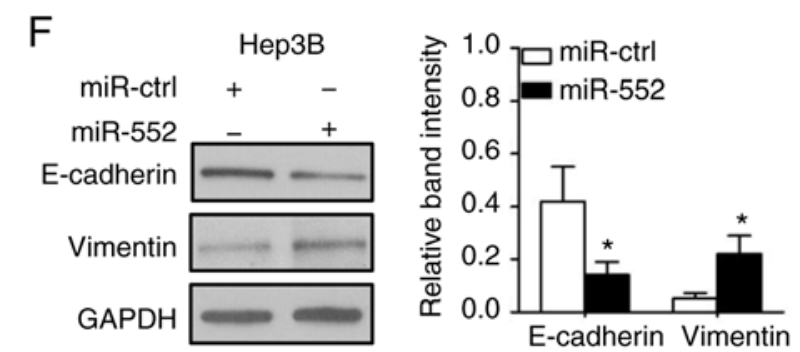

Figure 3. miR-552 promotes cell migration, invasion and epithelial-mesenchymal transition in vitro. (A) The percentage of residual area and (B) the number of invaded cells were decreased in Huh7 cells transfected with miR-552 inhibitors. The upregulation of miR-552 increased cell (C) migration and (D) invasion in Hep3B cells. (E) Inhibition of miR-552 increased E-cadherin expression but decreased vimentin expression in Huh-7 cells. (F) Decreased expression of E-cadherin and increased expression of vimentin was detected in Hep3B cells transfected with miR-552 mimics. ${ }^{*} \mathrm{P}<0.05$. miR, microRNA; E-cadherin, epithelial cadherin; ctrl, control; inhi, inhibitor.

WIF1 is a downstream target of miR-552 in HCC. miRNAs may bind to the 3'-UTR and act as inhibitors to decrease certain mRNAs expression. A total of 2 online MicroRNA targets prediction tools, TargetScan (http://www.targetscan. org/) and MiRanda (http://www.microrna.org), were used to predict WIF1 as a downstream target of miR-552. As indicated in Fig. 4A, knockdown of miR-552 increased WIF1 mRNA and protein expression in Huh-7 cells $(\mathrm{P}<0.05)$; conversely, miR-552 overexpression decreased WIF1 expression in Hep3B cells $(\mathrm{P}<0.05)$. Additionally, the wild-type and mutant-type dual-luciferase reporters were constructed according the binding sequence between miR-552 and WIF1 3'-UTR (Fig. 4B). As demonstrated in Fig. 4C, the dual-luciferase reporter assay demonstrated that the downregulation of miR-552 increased the luciferase activity of wild-type WIF1 3'-UTR $(\mathrm{P}<0.05)$, whereas the upregulation of miR-552 decreased the luciferase activity of the wild-type WIF1 3'-UTR $(\mathrm{P}<0.05)$. In either miR-552 knockdown or induction, there was no significant change on the luciferase activity of mutant WIF1 3'-UTR. Furthermore, a negative correlation between miR-552 and WIF1 expression was confirmed by Spearman correlation analysis (Fig. 4D; $\mathrm{r}=-0.703$; $\mathrm{P}<0.001$ ). These data provided convincing evidence to suggest that WIF1 is a direct target of miR-552 in HCC.

Oncogenic functions of miR-552 in HCC cells are mediated by WIFl. To additionally investigate the underlying mechanisms of miR-552/WIF1 axis on tumor progression, WIF1 expression was first inhibited in miR-552 downregulated Huh-7 cells (Fig. 5A; $\mathrm{P}<0.05$ ). The wound-healing and Transwell assays were then repeated to indicate that WIF1 knockdown abrogated the inhibitory effects of migration, invasion and EMT induced by miR-552 downregulation (Fig. 5B and C; $\mathrm{P}<0.05$ ). Subsequently, the same experiments were used to demonstrate that WIF1 overexpression decreased the migration, invasion and EMT in miR-552-upregulated Hep3B cells (Fig. 5D-F; P<0.05). 

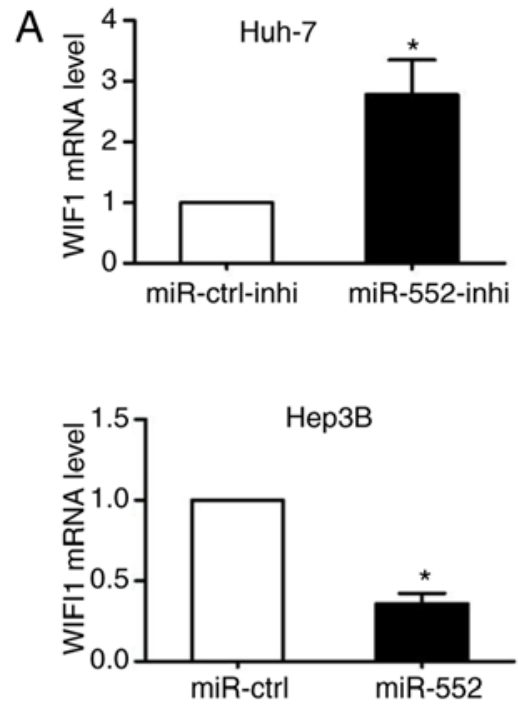
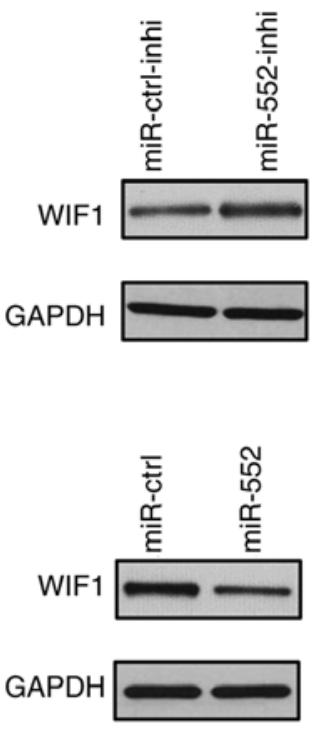
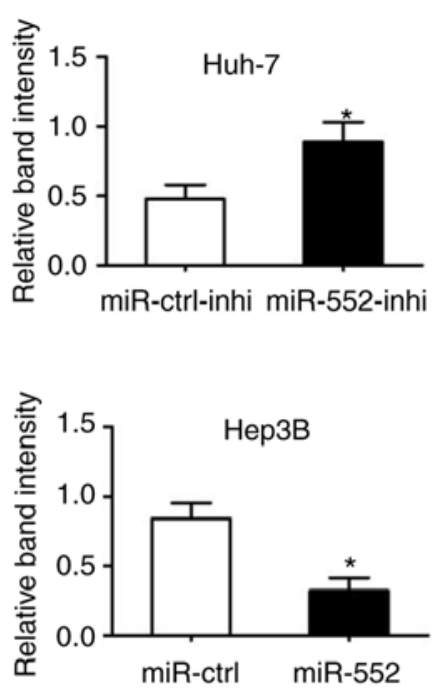

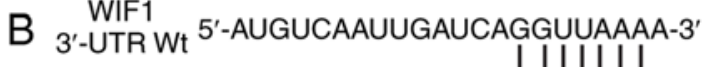 miR-552 3'-GGUUGUCCGUUUUCCAAUUUG-5' WIF1 3'-UTR Mt 5'-AUGUCAAUUGAUCACCUAUUUA-3'}
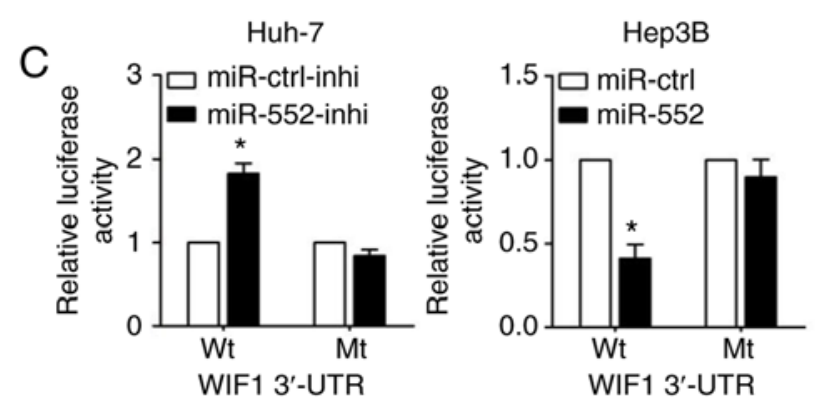
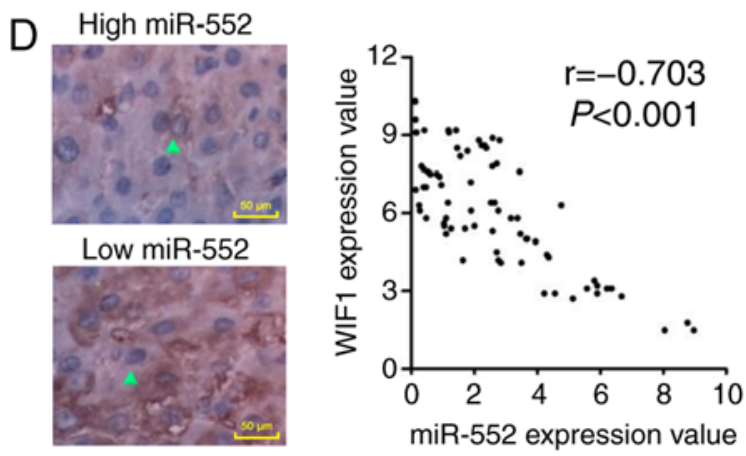

Figure 4. WIF1 is a downstream target of miR-552. (A) The mRNA and protein expression levels of WIF1 were upregulated in Huh-7 cells transfected with miR-552 inhibitors and downregulated in Hep3B cells transfected with miR-552 mimics. (B) The putative binding sites between miR-552 and WIF1 mRNA 3'-UTR was predicted by online databases. (C) The upregulation of miR-552 inhibited the luciferase activity that contained wild-type but not mutant-type 3'-UTR vectors of WIF1 in Huh-7 cells. Knockdown of miR-552 increased the luciferase activity of Hep3B cells that contained wild-type but not mutant-type 3'-UTR vectors of WIF1. (D) A negative correlation between miR-552 and WIF1 protein expression in HCC tissues was confirmed by Spearman correlation analysis. ${ }^{*} \mathrm{P}<0.05$. WIF1, Wnt inhibitory factor 1; miR, microRNA; UTR, untranslated region; Wt, wild-type; Mt, mutant; ctrl, control; inhi, inhibitor.

GSK3 $3 / \beta$-catenin signaling is crucial to the functions of miR-552 in HCC. Western blot analysis was used to demonstrate that miR-552 knockdown decreased GSK3 $\beta$ phosphorylation at Ser9 in Huh-7 cells (Fig. 6A; $\mathrm{P}<0.05$ ). The dephosphorylation of GSK3 $\beta$ inhibited $\beta$-catenin expression (Fig. 6A; $\mathrm{P}<0.05$ ). Conversely, miR-552 overexpression increased GSK3 $\beta$ phosphorylation and $\beta$-catenin expression in Hep3B cells (Fig. 6B; $\mathrm{P}<0.05$ ). Additionally, the inhibition of the GSK $3 \beta / \beta$-catenin signaling pathway by XAV939 (Fig. 6C; $\mathrm{P}<0.05$ ) abrogated the oncogenic functions of miR-552 overexpression on Hep3B cell migration and invasion (Fig. 6D and $\mathrm{E} ; \mathrm{P}<0.05$ ). XAV939 treatment also inhibited miR-552-induced EMT in Hep3B cells (Fig. 6F; $\mathrm{P}<0.05)$. Taken together, these data demonstrate that the oncogenic functions of miR-552 in HCC cells were attributed to a decreased activation of the GSK $3 \beta / \beta$-catenin signaling pathway, mediated by WIF1.

\section{Discussion}

The ectopic expressions of miRNAs in benign and malignant diseases may deregulate cell adhesion and movement functions. Anton et al (14) suggested that 2 adhesion associated genes, junctional adhesion molecule 1 and fascin actin-bundling protein 1, were inhibited following an overexpression of miR-143 and miR-145 in cervical cells. This abnormal regulation may destroy the cervical epithelial barrier, which is an important protector of preterm birth (15). A number of types of cancer (16), including HCC (17), also exhibited increased migration and invasion abilities caused by aberrant miRNA expression profiles, which are associated with cancer metastasis and poor prognosis.

Among these miRNAs, miR-552 was identified as an upregulated miRNA in colon cancer by principal component analysis (18). Notably, compared with the level of miR-552 

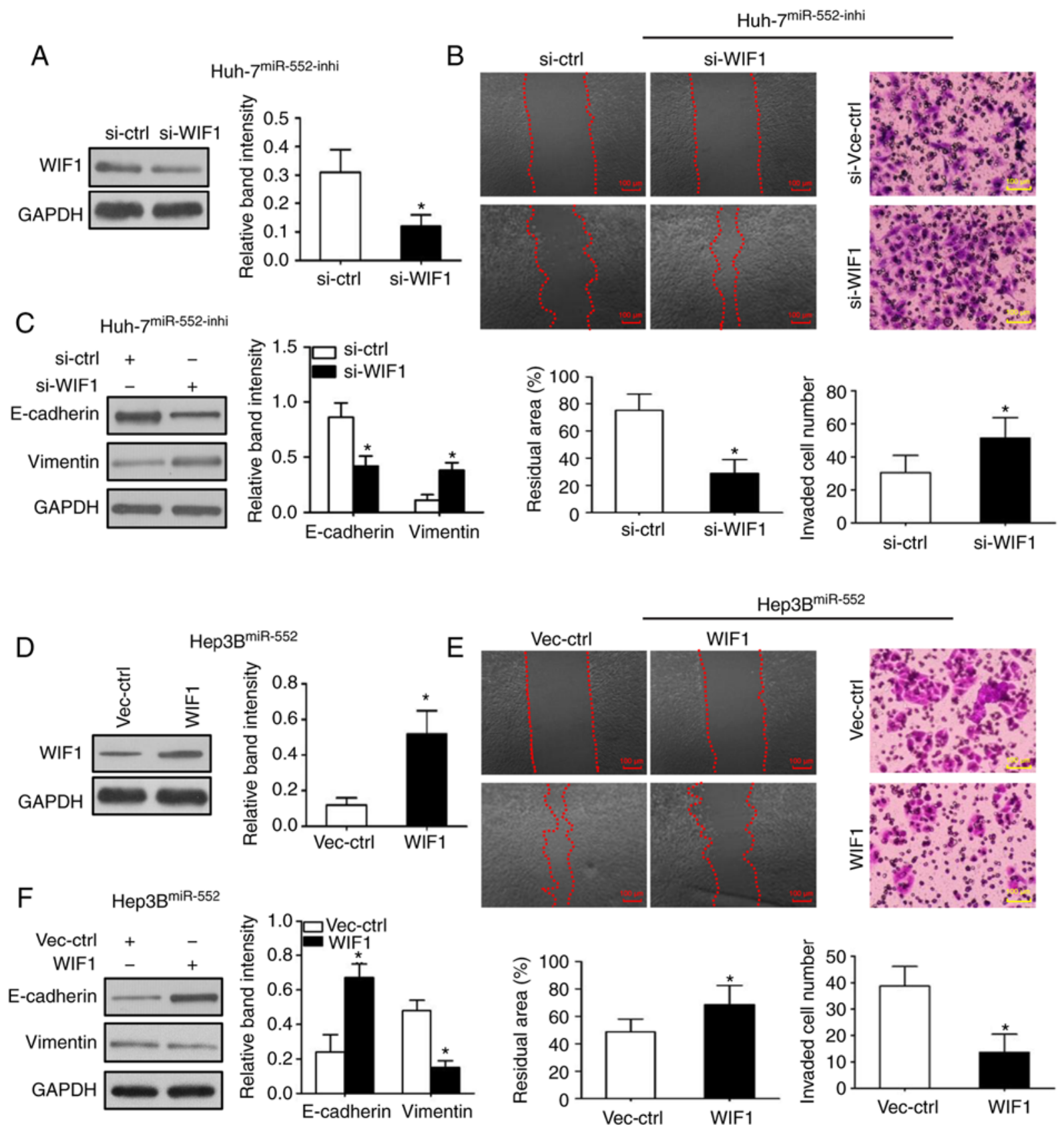

Figure 5. Altered expression of WIF1 abrogates the functions of miR-552 on hepatocellular carcinoma cells. (A) WIF1 expression was decreased by WIF1-specific siRNA transfection in Huh-7 cells expressing miR-552 inhibitors. (B) WIF1 knockdown promoted cell migration and invasion in Huh-7 cells expressing miR-552 inhibitors. (C) Inhibition of WIF1 decreased E-cadherin expression but increased vimentin expression in Huh-7 cells expressing miR-552 inhibitors. (D) WIF1 was overexpressed in Hep3B cells overexpressing miR-552. (E) Upregulation of WIF1 inhibited the migration and invasion in Hep3B cells overexpressing miR-552. (F) The upregulation of WIF1 increased E-cadherin expression but decreased vimentin expression in Hep3B cells overexpressing miR-552. "P<0.05. WIF1, Wnt inhibitory factor 1; miR, microRNA; ctrl, control; inhi, inhibitor; E-cadherin, epithelial cadherin; siRNA, small interfering RNA; Vec, vector.

in defective DNA mismatch repair (MMR) tumors, miR-552 expression is upregulated in proficient DNA MMR tumors (19). This indicates that miR-552 has potential to regulate colon cancer cell proliferation and invasion. In the present study, it was identified that miR-552 was increased in HCC tissues, particularly in tumors with portal vein invasion, lymph node metastasis and other features of aggressive behavior. The in vitro results of the present study also indicated that miR-552 may serve a pro-invasive role in HCC cells: The inhibition of miR-552 not only decreased migration and invasion of Huh-7 cells but also impaired the EMT markers' expression, whereas an overexpression of miR-552 increased the migration, invasion and EMT of Hep3B cells.

The Wnt/ $\beta$-catenin pathway involves a family of proteins that is critical to a number of biological functions including cell differentiation (20) and organogenesis (21). In HCC, the Wnt/ $\beta$-catenin pathway has been demonstrated to promote cell proliferation, invasion and angiogenesis (22). The WIF1 gene, located on human chromosome $12 q 14$, encodes a secreted protein that inactivates the Wnt/ $\beta$-catenin signaling pathway by binding to Wnt proteins (23). In the present study, qPCR, western blot analysis and dual-luciferase reporter 

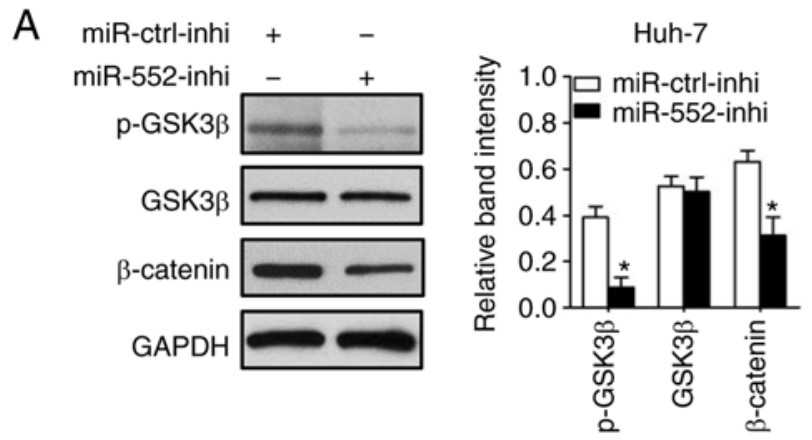
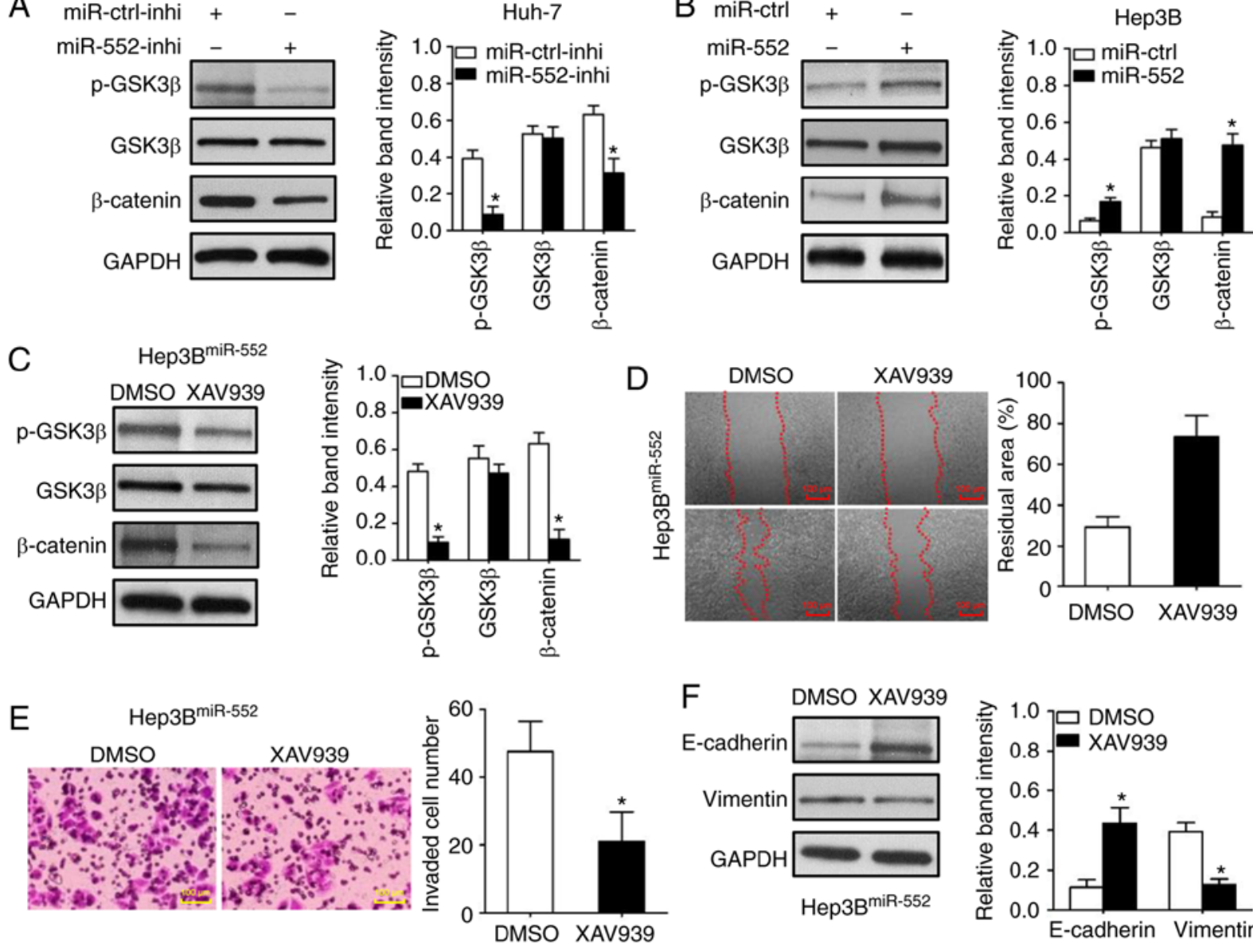
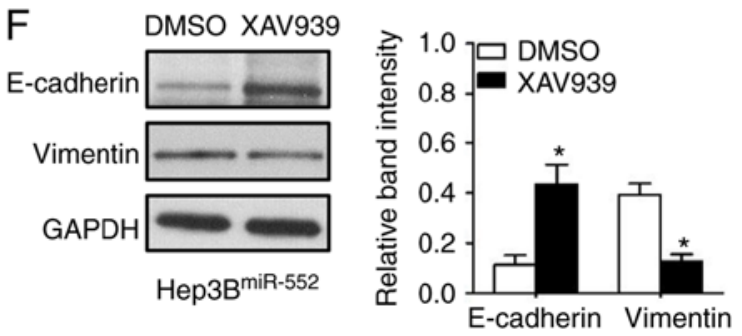

Figure 6. Pro-invasive functions of miR-552 are achieved through regulating the GSK3 $\beta / \beta$-catenin signaling pathway. Phosphorylation of GSK3 $\beta$ at Ser9 and total expression levels of $\beta$-catenin were decreased in (A) Huh7 cells transfected with miR-552 inhibitors, but increased in (B) Hep3B cells transfected with miR-552 mimics. (C) XAV939 treatment decreased GSK3 $\beta$ phosphorylation and $\beta$-catenin expression in Hep3B cells overexpressing miR-552. XAV939 treatment inhibited cell (D) migration and (E) invasion in Hep3B cells overexpressing miR-552. (F) XAV939 treatment increased E-cadherin expression

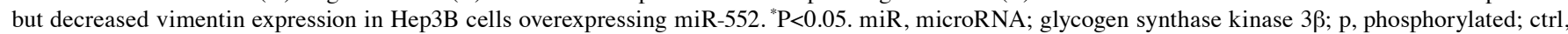
control; inhi, inhibitor; DMSO, dimethyl sulfoxide; E-cadherin, epithelial cadherin.

assay methods were used to confirm that WIF1 is a downstream target of miR-552. The clinical analysis also revealed an inverse correlation between miR-552 and WIF1 protein expression in HCC tissues. As miR-552 binds to the 3'-UTR of WIF1 mRNA to form the RNA-induced silencing complex (RISC), the miR-552/WIF1 RISC may lead to the degradation of WIF1 mRNA and arrest the subsequent translation process. Therefore, miR-552 may downregulate WIF1 mRNA and protein expression. As a critical Wnt antagonist, WIF1 is involved in the suppression of invasion in various cancer cell lines (24). Similarly, the present study identified that the altered expression of WIF1 abrogated the pro-invasive functions of miR-552 on HCC cells. As WIF1 may abolish the binding of the Wnt ligand to the LRP-5/6 receptors (25), we hypothesized whether miR-552 knockdown may cause the dephosphorylation of GSK3 $\beta$ at Ser9. As expected, miR-552 knockdown decreased GSK $3 \beta$ phosphorylation and $\beta$-catenin expression. XAV939 has been previously confirmed as an inhibitor of $\beta$-catenin (26). In the present study, XAV939 was used to treat Hep3B cells with miR-552 overexpression. The present study demonstrated that XAV939 also inhibited the cell migration, invasion and EMT processes induced by miR-552. As a transcription factor, $\beta$-catenin may increase the expression of a number of EMT-associated transcription factors (27), including zinc finger protein SNAI1, zinc finger protein SNAI2 and twist-related protein 1. This may explain why miR-552 exhibits pro-invasive functions in HCC.

In conclusion, miR-552 is highly associated with HCC malignancies and has potential value to be a prognostic biomarker for patients with HCC.

\section{Acknowledgements}

Not applicable.

\section{Funding}

The present study was supported by a grant from the National Natural Scientific Foundation of China (grant no. 81472247).

\section{Availability of data and materials}

The analyzed datasets generated during the study are available from the corresponding author on reasonable request. 


\section{Authors' contributions}

CLi contributed to the design of the study and wrote the manuscript. ZW, SC, JZ and KQ performed the study; and CLiu contributed to the design of the study. All authors read and approved the final manuscript.

\section{Ethics approval and consent to participate}

The use of clinical samples was approved by the Ethics Committee of the First Affiliated Hospital of Xi'an Jiaotong University. Written informed consent was obtained from all patients enrolled in the present study.

\section{Patient consent for publication}

Written informed consent was obtained and signed by each patient enrolled in the present study.

\section{Competing interests}

The authors declare that they have no competing interests.

\section{References}

1. Fang LL, Wang XH, Sun BF, Zhang XD, Zhu XH, Yu ZJ and Luo $\mathrm{H}$ : Expression, regulation and mechanism of action of the miR-17-92 cluster in tumor cells (Review). Int J Mol Med 40 1624-1630, 2017.

2. Liu H, Lei C, He Q, Pan Z, Xiao D and Tao Y: Nuclear functions of mammalian MicroRNAs in gene regulation, immunity and cancer. Mol cancer 17: 64, 2018.

3. Tang A, Hallouch O, Chernyak V, Kamaya A and Sirlin CB: Epidemiology of hepatocellular carcinoma: target population for surveillance and diagnosis. Abdom Radiol 43: 13-25, 2018.

4. Yegin EG, Oymaci E, Karatay E and Coker A: Progress in surgical and nonsurgical approaches for hepatocellular carcinoma treatment. Hepatobiliary Pancreat Dis Int 15: 234-256, 2016.

5. Tian Q, Xiao Y, Wu Y, Liu Y, Song Z, Gao W, Zhang J, Yang J, Zhang Y, Guo T, et al: MicroRNA-33b suppresses the proliferation and metastasis of hepatocellular carcinoma cells through the inhibition of Sal-like protein 4 expression. Int J Mol Med 38: $1587-1595,2016$.

6. Wu WL, Wang WY, Yao WQ and Li GD: Suppressive effects of microRNA-16 on the proliferation, invasion and metastasis of hepatocellular carcinoma cells. Int J Mol Med 36: 1713-1719, 2015.

7. Li C, Jiang Y, Miao R, Qu K, Zhang J and Liu C: MicroRNA-1271 functions as a metastasis and epithelial-mesenchymal transition inhibitor in human HCC by targeting the PTP4A1/c-Src axis. Int J Oncol 52: 536-546, 2018.

8. Xia ZS, Wang L, Yu T, Zhong W, Lian GD, Wu D, Zhou HM and Chen GC: MiR-5000-3p, miR-5009-3P and miR-552: potential microRNA biomarkers of side population cells in colon cancer Oncol Rep 32: 589-596, 2014.

9. Wang J, Li H, Wang Y, Wang L, Yan X, Zhang D, Ma X, Du Y, Liu X and Yang Y: MicroRNA-552 enhances metastatic capacity of colorectal cancer cells by targeting a disintegrin and metalloprotease 28. Oncotarget 7: 70194-70210, 2016.

10. Brabletz T, Kalluri R, Nieto MA and Weinberg RA: EMT in cancer. Nat Rev Cancer 18: 128-134, 2018.

11. Livak and Schmittgen: Analysis of relative gene expression data using real-time quantitative PCR and the $2-\Delta \Delta \mathrm{Ct}$ method Methods 25: 402-408, 2001.
12. Li C, Jiang Y, Miao R, Qu K, Zhang J and Liu C: MicroRNA-1271 functions as a metastasis and epithelial-mesenchymal transition inhibitor in human HCC by targeting the PTP4A1/c-Src axis. Int J Oncol 52: 536-546, 2018.

13. Duseja A: Staging of hepatocellular carcinoma. J Clin Exp Hepatol 4: S74-S79, 2014.

14. Anton L, DeVine A, Sierra LJ, Brown AG and Elovitz MA: miR-143 and miR-145 disrupt the cervical epithelial barrier through dysregulation of cell adhesion, apoptosis and proliferation. Sci Rep 7: 3020, 2017.

15. Akgul Y, Word RA, Ensign LM, Yamaguchi Y, Lydon J, Hanes J and Mahendroo M: Hyaluronan in cervical epithelia protects against infection-mediated preterm birth. J Clin Invest 124: 5481-5489, 2014

16. Lou W, Liu J, Gao Y, Zhong G, Chen D, Shen J, Bao C, Xu L, Pan J, Cheng J, et al: MicroRNAs in cancer metastasis and angiogenesis. Oncotarget 8: 115787-115802, 2017.

17. Zhang Y, Wei Y, Li X, Liang X, Wang L, Song J, Zhang X, Zhang C, Niu J, Zhang P, et al: microRNA-874 suppresses tumor proliferation and metastasis in hepatocellular carcinoma by targeting the DOR/EGFR/ERK pathway. Cell Death Dis 9: 130, 2018.

18. Wang XY, Wu MH, Liu F, Li Y, Li N, Li GY and Shen SR: Differential miRNA expression and their target genes between NGX6-positive and negative colon cancer cells. Mol Cell Biochem 345: 283-290, 2010.

19. Oberg AL, French AJ, Sarver AL, Subramanian S, Morlan BW, Riska SM, Borralho PM, Cunningham JM, Boardman LA, Wang L, et al: miRNA expression in colon polyps provides evidence for a multihit model of colon cancer. PLoS One 6: e20465, 2011

20. Cui YM, Han XH, Lin YY, Lv WW and Wang YL: TNF-alpha was involved in calcium hydroxide-promoted osteogenic differentiation of human DPSCs through NF-kappaB/p38MAPK/Wnt pathway. Die Pharmazie 72: 329-333, 2017.

21. Rankin SA, McCracken KW, Luedeke DM, Han L, Wells JM, Shannon JM and Zorn AM: Timing is everything: Reiterative Wnt, BMP and RA signaling regulate developmental competence during endoderm organogenesis. Dev Biol 434: 121-132, 2018.

22. Chen J, Rajasekaran M and Hui KM: Atypical regulators of Wnt/beta-catenin signaling as potential therapeutic targets in hepatocellular carcinoma. Exp Biol Med 242: 1142-1149, 2017.

23. Zheng Y, Li X, Jiang Y, Xu Y, Song B, Zhou Q, Liang X and Yang X: Promoter hypermethylation of Wnt inhibitory factor-1 in patients with lung cancer: A systematic meta-analysis. Medicine 95: e5433, 2016.

24. GuoH,Zhou S, Tan L, Wu X, WuZ and RanR: Clinicopathological significance of WIF1 hypermethylation in NSCLC, a meta-analysis and literature review. Oncotarget 8: 2550-2557, 2017.

25. Schmid SC, Sathe A, Guerth F, Seitz AK, Heck MM, Maurer T, Schwarzenböck SM, Krause BJ, Schulz WA, Stoehr R, et al: Wntless promotes bladder cancer growth and acts synergistically as a molecular target in combination with cisplatin. Urol Oncol 35: 544.e1-544.e10, 2017.

26. Guo W, Shen F, Xiao W, Chen J and Pan F: Wnt inhibitor XAV939 suppresses the viability of small cell lung cancer NCI-H446 cells and induces apoptosis. Oncol Lett 14: 6585-6591, 2017.

27. Wu C, Zhuang Y, Jiang S, Liu S, Zhou J, Wu J, Teng Y, Xia B, Wang $\mathrm{R}$ and Zou X: Interaction between Wnt/beta-catenin pathway and microRNAs regulates epithelial-mesenchymal transition in gastric cancer (Review). Int J Oncol 48: 2236-2246, 2016.

This work is licensed under a Creative Commons Attribution-NonCommercial-NoDerivatives 4.0 International (CC BY-NC-ND 4.0) License. 